\title{
New Element Models in Discrete Structural Analysis
}

\author{
by Tadahiko Kawai*
}

\begin{abstract}
Summary
A family of new element models in discrete structural analysis is proposed in this paper.

These models consist of finite number of small rigid bodies connected with springs distributed over the contact area of two neighbouring bodies. In general size of stiffness matrices of these elements are at most $(6 \times 6)$ which are equal to or even smaller than $1 / 2$ of those of conventional finite elements so that considerable reduction of computing time can be expected.

Effectiveness of these elements in nonlinear structural analysis will be demonstrated by several numerical examples.
\end{abstract}

\section{Theoretical Basis of New Element Models}

Recently the present author proposed new physical models for beam and plate bending problems in order to reduce computing time especially in nonlinear analysis ${ }^{1} * *$, and shortly after the same idea has been extended to analysis of the plane strain and plane stress problems.

In what follows derivation of new elements for analysis of beam bending, plate bending and plane strain problems will be briefly described.

First of all, theoretical basis of new element models is explained briefly. Consider the bending problem of a beam under lateral loads. Within elastic range of deformation, deformation is distributed throughout the beam, but once plastic deformation starts either at the point of load application or at the beam ends, elastic deformation will be absorbed in the narrow portion of a beam where plastic deformation takes place and at the ultimate stage of loading a number of the so-called the "plastic hinge" will be formed so that the beam structure will collapse just like a link mechanism.

This mechanism consists of rigid bars and plastic hinges.

In case of bending problems of concrete slabs, similar experimental evidence will be observed.

That is, within the range of elastic bending, deformation is distributed over the whole plate area, however, at the limiting stage of load application the plate will collapse under a certain mechanism

\footnotetext{
* Institute of Industrial Science, University of Tokyo

** The number in bracket indicates the number of literature at the end of this paper.
}

which consists of rigid plate segments, and plastic hinge lines connecting those plate segments. The so-called "slip line theory" is also well known in plane stress as well as plane strain problems in the theory of plasticity. ${ }^{2)-8)}$

According to this theory, it is assumed that two dimensional solids will move under a certain mechanism which consists of two dimensional rigid segments and slip lines connecting rigid segments and along which they allow the relative sliding of two neighboring segments. From observation of these three experimental evidence, the present author conceived concept of the following "Rigid Bodies-Spring Models".

(i) Beam bending element

Consider deformation of two rigid bars connected by one rotational spring as shown in Fig. 1 . It is assumed that two bars are displaced as shown in this figure under some lateral loading.

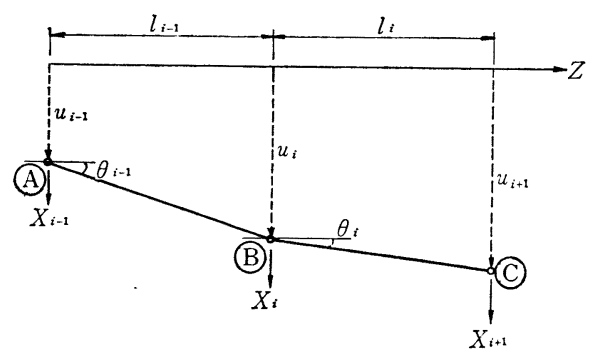

Fig. 1 A new beam bending element

Denoting the displacements of the point A, B and C by $u_{i-1}, u_{i}, u_{i+1}$, the following strain energy expression can be derived easily 


$$
V\left(u_{i-1}, u_{i}, u_{i+1}\right)=\frac{k_{b}}{2}\left\{\left(\frac{u_{i+1}-u_{i}}{l_{i}}\right)^{2}-\left(\frac{u_{i}-u_{i-1}}{l_{i-1}}\right)^{2}\right\}
$$

where $k_{b}$ is a spring constant.

It is not difficult to derive the following stiffness matrix of a given system by applying Castigliano's Theorem

$\left\{\begin{array}{l}X_{i-1} \\ X_{i} \\ X_{i+1}\end{array}\right\}=k_{b}\left(\begin{array}{cc}\frac{1}{l_{i-1}^{2}} & \text { SYM. } \\ -\frac{1}{l_{i-1}}\left(\frac{1}{l_{i}}+\frac{1}{l_{i-1}}\right)\left(\frac{1}{l_{i-1}}+\frac{1}{l_{i}}\right)^{2} \\ \frac{1}{l_{i-1} l_{i}}-\frac{1}{l_{i}}\left(\frac{1}{l_{i}}+\frac{1}{l_{i-1}}\right) \frac{1}{l_{i}^{2}}\end{array}\right\}\left\{\begin{array}{l}u_{i-1} \\ u_{i} \\ u_{i+1}\end{array}\right\}$

$M_{i}=k_{b}\left\lfloor\frac{1}{l_{i-1}},-\left(\frac{1}{l_{i-1}}+\frac{1}{l_{i}}\right), \frac{1}{l_{i}}\right\rfloor\left\{\begin{array}{l}u_{i-1} \\ u_{i} \\ u_{i+1}\end{array}\right\}$

It can be seen from eq. (2) that the size of this matrix is $2 \times 2$ for each bar element and it is $1 / 2$ of that of the conventional beam bending element.

(ii) Plate bending element

Consider assemblage of rigid plate elements shown in Fig. 2. Triangular plates $\Delta 012$ and $\Delta 023$ are

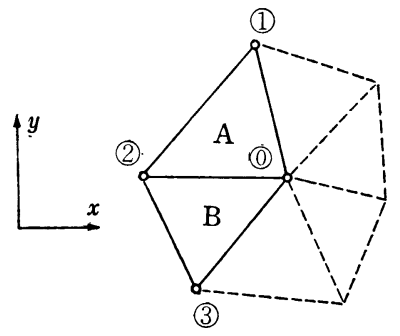

Fig. 2 A new plate bending element

connected by a spring whose constant is $k_{p}$. Before loading these plates were on the $x, y$ plane and under a given loading they are displaced to the position whose equation is given by the following equation

$$
l_{A} x+m_{A} y+n_{A} z=p_{A}
$$

Denoting lateral displacement of the point (0) $\left(x_{0}, y_{0}\right)$, (1) $\left(x_{1}, y_{1}\right)$, (2) $\left(x_{2}, y_{2}\right)$ by $w_{0}, w_{1}$, and $w_{2}$ respectively, direction cosines $\left(l_{A}, m_{A}, n_{A}\right)$ and $p_{A}$ can be expressed as follows:

$$
\left.\begin{array}{ll}
l_{A}= \pm \frac{\Delta_{11}}{\sqrt{\Delta_{11}^{2}+\Delta_{12}^{2}+\Delta_{10}^{2}}}, & m_{A}= \pm \frac{\Delta_{12}}{\sqrt{\Delta_{11}^{2}+\Delta_{12}^{2}+\Delta_{13}^{2}}} \\
n_{A}= \pm \frac{\Delta_{10}}{\sqrt{\Delta_{11}^{2}+\Delta_{12}^{2}+\Delta_{10}^{2}}}, & p_{A}= \pm \frac{x_{0} \Delta_{11}+y_{0} \Delta_{12}+w_{0} \Delta_{10}}{\sqrt{\Delta_{11}^{2}+\Delta_{12}^{2}+\Delta_{13}^{2}}}
\end{array}\right\}
$$

where

$$
\Delta_{10}=\left|\begin{array}{ll}
x_{01} & y_{01} \\
x_{12} & y_{12}
\end{array}\right| \quad \Delta_{11}=-\left|\begin{array}{ll}
w_{01} & y_{01} \\
w_{12} & y_{12}
\end{array}\right| \quad \Delta_{12}=-\left|\begin{array}{ll}
x_{01} & w_{01} \\
x_{12} & w_{12}
\end{array}\right|
$$

The similar expressions for $\Delta 023$ can be drived. In the deformed state $\Delta 012$ and $\Delta 023$ are inclined each other through the rotation angle $\theta_{A B}$ and the following relation can be easily obtained

$$
\cos \theta_{A B} \fallingdotseq 1-\frac{\theta_{A B}^{2}}{2}=l_{A} l_{B}+m_{A} m_{B}+n_{A} n_{B}
$$

When $\theta_{A B}$ is small, the strain energy stored in the connection spring will be given as follows:

$$
\begin{aligned}
V(\boldsymbol{w})=\frac{1}{2} k_{p} \theta_{A B}^{2}= & -\frac{1}{\Delta_{10} \Delta_{20}}\left(\Delta_{11} \Delta_{12}+\Delta_{12} \Delta_{22}\right) \\
& +\frac{1}{2 \Delta_{10}^{2}}\left(\Delta_{11}^{2}+\Delta_{12}^{2}\right)+\frac{1}{2 \Delta_{20}^{2}}\left(\Delta_{21}^{2}+\Delta_{22}^{2}\right)
\end{aligned}
$$

where $\boldsymbol{w}^{T}=\left\lfloor w_{0}, w_{1}, w_{2}, w_{3}\right\rfloor$

Applying Castigliano's theorem, the following reaction force vecter $\boldsymbol{R}$ can be derived

$$
R=\frac{\partial \nu}{\partial w}=K w
$$

where $K$ is the stiffness matrix to be obtained. The final form of the stiffness matrix is shown in the Table 1 .

(iii) A physical model in plane strain problem

Consider two rigid triangular plates which are connected by three different types of springs $k_{d}, k_{s}$ and $k_{r}$ at the middle point of boundary edges as shown in Fig. 3. Centroidal displacements of each plate is denoted by $\left(u_{1}, v_{2}, \theta_{1}\right)$ and $\left(u_{2}, v_{2}, \theta_{2}\right)$ respectively.

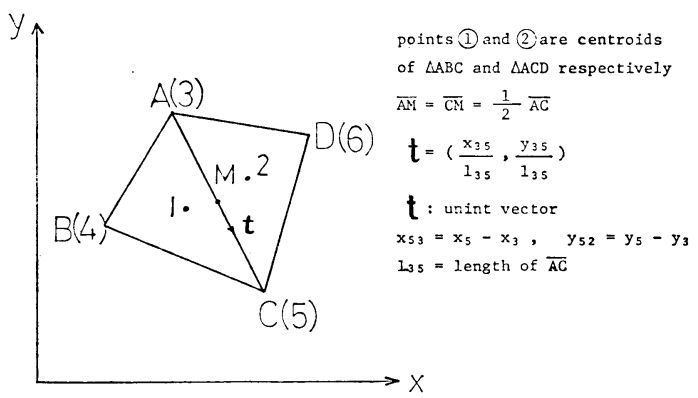

Fig. 3 A new plane strain element

The displacements of an arbitrary point in $\triangle \mathrm{ABC}$, for example, can be given by the following equation

$$
\left.\begin{array}{l}
u=u_{1}+\left(y-y_{1}\right) \theta_{1} \\
v=v_{1}-\left(x-x_{1}\right) \theta_{1}
\end{array}\right\}
$$

where the rotational displacement $\theta_{1}$ is assumed to be very small.

After some loading, two plates are displaced to positions as shown in Fig. 4. The relative displacement of the edge $\overline{\mathrm{AC}}$ in each plate is given by $\delta_{V}$, $\delta_{H}$ and $\varphi$. From simple consideration of geometry, the relative displacement vector of the middle point $M$ on the edge $\overline{\mathrm{AC}}, \overrightarrow{M^{\prime} M^{\prime \prime}}$ can be given by the following equation 
Table 1 Stiffness matrix of a new plate bending element $\left(\times \frac{k_{A B}}{\Delta_{10} \Delta_{20}}\right)$

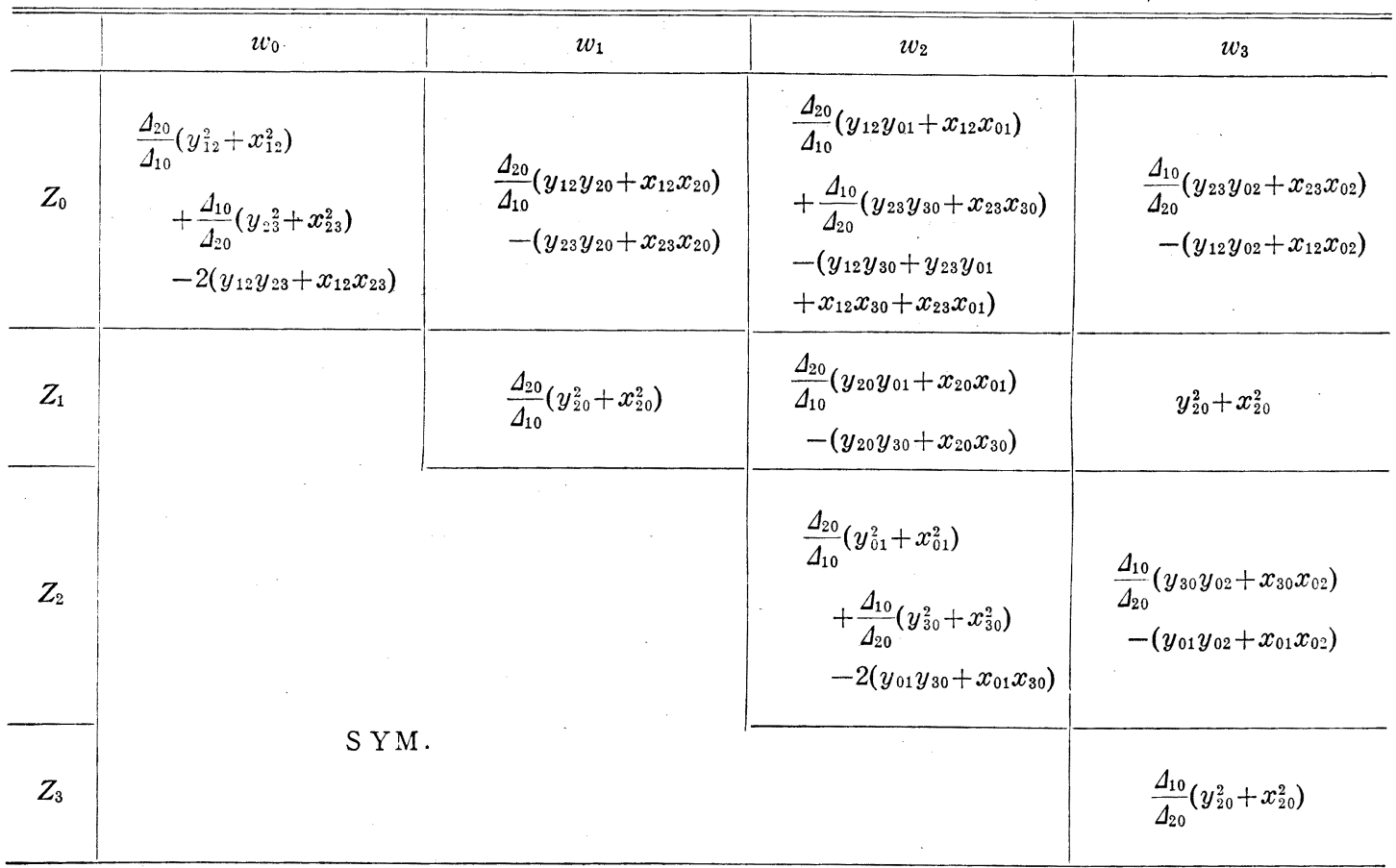

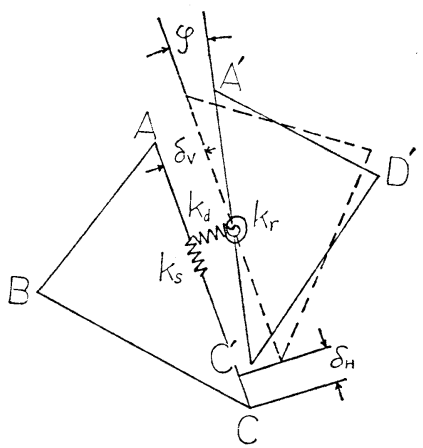

Fig. 4 Two plates' positions after deformation

$$
\left.\begin{array}{l}
\left(\overrightarrow{M^{\prime} M^{\prime \prime}}\right)_{x}=\frac{1}{2}\left\{2 u_{21}+\left(y_{32}+y_{52}\right) \theta_{2}-\left(y_{31}+y_{51}\right) \theta_{1}\right\} \\
\left(\overrightarrow{M^{\prime} M^{\prime \prime}}\right)_{y}=\frac{1}{2}\left\{2 v_{21}-\left(x_{32}+y_{52}\right) \theta_{2}+\left(x_{31}+x_{51}\right) \theta_{1}\right\}
\end{array}\right\}
$$

where

$$
u_{i j}=u_{i}-u_{j}, \quad v_{i j}=v_{i}-v_{j}
$$

Denoting an unit vector along the edge $\overline{\mathrm{AC}}$ by $\boldsymbol{t}$ before deformation as shown in Fig. 3 , the displacement component $\delta_{H}$ of the vector $\overrightarrow{M^{\prime} M^{\prime \prime}}$ along the edge $\overline{\mathrm{AC}}$ can be given as follows:

$$
\begin{aligned}
\delta_{H} \fallingdotseq\left(\overrightarrow{M^{\prime} M^{\prime \prime}}, \boldsymbol{t}\right)= & \frac{1}{2 l_{35}}\left[x _ { 5 3 } \left\{2 u_{21}+\left(y_{32}+y_{53}\right) \theta_{2}\right.\right. \\
& \left.-\left(y_{31}+y_{51}\right) \theta_{1}\right\}+y_{53}\left\{2 v_{21}\right. \\
& \left.\left.-\left(x_{32}+x_{52}\right) \theta_{2}+\left(x_{31}+x_{51}\right) \theta_{1}\right\}\right]
\end{aligned}
$$

where $l_{35}$ is length of the edge $\overline{\mathrm{AC}}$ before deformation. Similarly the displacement $\delta_{V}$ component of the vector $\overrightarrow{M^{\prime} M^{\prime \prime}}$ perpendicular to the edge $\overline{\mathrm{AC}}$ can be given by the following equation

$$
\begin{aligned}
\delta_{V}^{2} \fallingdotseq\left|\boldsymbol{t} \times \overrightarrow{M^{\prime} M^{\prime \prime}}\right|^{2}= & \frac{1}{4 l_{35}^{2}}\left[x _ { 5 3 } \left\{2 v_{21}-\left(x_{32}-x_{52}\right) \theta_{2}\right.\right. \\
& \left.+\left(x_{31}+x_{51}\right) \theta_{1}\right\}-y_{52}\left\{2 u_{21}\right. \\
& \left.\left.+\left(y_{32}+y_{52}\right) \theta_{2}-\left(y_{31}+y_{51}\right) \theta_{1}\right\}\right]^{2}
\end{aligned}
$$

Relative angle change $\varphi$ of the edges $\overline{\mathrm{AC}}$ and $\overline{\mathrm{A}^{\prime} \mathrm{C}^{\prime}}$ is also obtained from following equation

$$
\cos \varphi=\left(t, t^{\prime}\right) \fallingdotseq 1-\frac{\varphi^{2}}{2}
$$

where $\boldsymbol{t}^{\prime}$ is the unit vector along the edge $\overline{\mathrm{A}^{\prime} \mathrm{C}^{\prime}}$ after deformation.

$$
\begin{aligned}
\therefore \quad \frac{\varphi^{2}}{2}= & \frac{1}{l_{35}^{2}}\left[\left(u_{53}+u_{53}^{\prime}\right) x_{53}+\left(v_{53}+v_{53}^{\prime}\right) y_{53}\right. \\
& \left.+u_{53} u_{53}^{\prime}+v_{53} v_{53}^{\prime}\right]
\end{aligned}
$$

Now strain energy $V$ to be stored in the spring $k_{d}, k_{s}$ and $k_{r}$ after deformation will be given as follows:

$$
V=\frac{1}{2} k_{d} \delta_{V}^{2}+\frac{1}{2} k_{s} \delta_{H}^{2}+\frac{1}{2} k_{r} \varphi^{2}
$$

In view of (13), (14), (16) and (17) it is clearly seen that the strain energy $V$ is a quadratic function of $\left(u_{1}, v_{1}, \theta_{1}\right)$ and $\left(u_{2}, v_{2}, \theta\right)$ and therefore applying Castigliano's theorem again, the stiffness matrix for analysis of plane strain problems can be derived. 
Table 2 Stiffness matrix of a new plane strain element $\left(\times 1 / l_{35}^{2}\right)$

\begin{tabular}{|c|c|c|c|c|c|c|}
\hline & $u_{1}$ & $v_{1}$ & $\theta_{1}$ & $u_{2}$ & $v_{2}$ & $\theta_{2}$ \\
\hline$X_{1}$ & $k_{d} y_{53}^{2}+y_{s} x_{53}^{2}$ & & & \multirow{3}{*}{\multicolumn{3}{|c|}{$\left.\begin{array}{l}2 \Delta_{11}=x_{53}\left(x_{31}+x_{51}\right)+y_{53}\left(y_{31}+y_{51}\right) \\
2 \Delta_{12}=x_{53}\left(y_{32}+y_{52}\right)-y_{53}\left(x_{32}+x_{52}\right) \\
2 \Delta_{21}=-x_{53}\left(y_{31}+y_{51}\right)+y_{53}\left(x_{31}+x_{51}\right) \\
2 \Delta_{22}=-x_{53}\left(x_{32}+x_{52}\right)-y_{53}\left(y_{32}+y_{52}\right)\end{array}\right\}$}} \\
\hline$Y_{1}$ & $-\left(k_{d}-k_{s}\right) x_{53} y_{53}$ & $k_{a} x_{53}^{2}+k_{s} k_{53}^{2}$ & & & & \\
\hline$M_{1}$ & $\begin{array}{l}k_{d} y_{53} \Delta_{11} \\
\quad-k_{\boldsymbol{\delta}} x_{53} \Delta_{21}\end{array}$ & $\begin{array}{l}-\left(k_{a} x_{53} \Delta_{11}\right. \\
\left.\quad+k_{s} y_{53} \Delta_{21}\right)\end{array}$ & $\begin{array}{l}k_{d} \Delta_{11}^{2}+k_{s} \Delta_{21}^{2} \\
\quad+k_{r} l_{35}^{2}\end{array}$ & & & \\
\hline$X_{2}$ & $-\left(k_{d} y_{53}^{2}+k_{s} x_{53}^{2}\right)$ & $\left(k_{d}-k_{s}\right) x_{53} y_{53}$ & $\begin{array}{l}-\left(k_{d} y_{53} \Delta_{11}\right. \\
\left.-k_{s} x_{53} \Delta_{21}\right)\end{array}$ & $k_{a} y_{53}^{2}+k_{s} x_{53}^{2}$ & & \\
\hline$Y_{2}$ & $\left(h_{d}-k_{s}\right) x_{53} y_{53}$ & $-\left(k_{d} x_{53}^{2}+k_{s} y_{53}^{2}\right)$ & $\begin{array}{l}k_{a} x_{53} \Delta_{11} \\
\quad+k_{s} y_{53} \Delta_{21}\end{array}$ & $-\left(k_{d}-k_{s}\right) x_{53} y_{53}$ & $k_{d} x_{53}^{2}+k_{s} y_{53}^{2}$ & \\
\hline$M_{2}$ & $\begin{array}{l}k_{d} y_{53} \Delta_{22} \\
\quad-k_{s} x_{53} \Delta_{12}\end{array}$ & $\begin{array}{l}-\left(k_{a} x_{53} \Delta_{22}\right. \\
\left.\quad+k_{s} y_{53} \Delta_{12}\right)\end{array}$ & $\begin{array}{l}k_{a} \Delta_{11} \Delta_{22}+k_{s} \Delta_{21} \Delta_{12} \\
\quad-k_{r} l_{35}^{2}\end{array}$ & $\begin{array}{l}-\left(k_{a} y_{53} \Delta_{22}\right. \\
\left.-k_{s} x_{53} \Delta_{12}\right)\end{array}$ & $\begin{array}{l}k_{d} x_{53} \Delta_{22} \\
\quad+k_{s} y_{53} \Delta_{12}\end{array}$ & $\begin{array}{l}k_{d} \Delta_{22}^{2}+k_{s} \Delta_{12}^{2} \\
\quad+k_{r} l_{35}^{2}\end{array}$ \\
\hline
\end{tabular}

The final form of the stiffness matrix is given in the Table 2 .

(iv) Method of determination of the spring constants

The spring constants in these stiffness matrices can be determined theoretically as follows:

In case of a beam element (Fig. 1), for example, the curvature $r$ can be expressed by

$$
r=\frac{2}{l_{i}+l_{i-1}}\left(\theta_{i}-\theta_{i-1}\right)
$$

From the moment-curvature relation the following equation is derived

$$
M=k_{b}\left(\theta_{i}-\theta_{i-1}\right)=E I r
$$

Substituting eq. (18) into eq. (19) the following relation is easily obtained

$$
k_{b}=\frac{2 E I}{l_{i}+l_{i-1}}
$$

It is not difficult to derive the following formula for the spring constant of a given plate element as shown in Fig. 5.

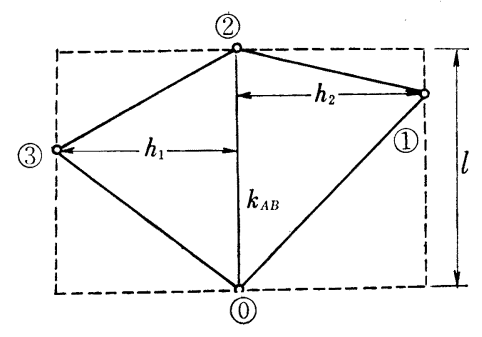

Fig. 5 Determination of the spring constant in the plate bending element

$$
k_{p}=\frac{2 D l}{h_{1}+h_{2}}
$$

On the other hand spring constants $k_{d}, k_{s}$ and $k_{r}$ in the plane strain problems can be determined in

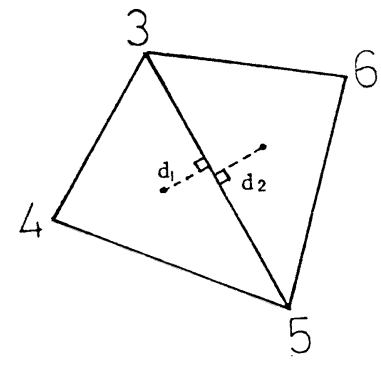

Fig. 6 Determination of the spring constants in the plane strain element

the following way. Considering two plates shown in Fig. 6 the normal strains $\varepsilon$ and shearing strain $\gamma$ in these springs may be given as follows:

$$
\left.\begin{array}{l}
\varepsilon=\frac{\delta_{V}}{d_{1}+d_{2}}=\frac{(1+\nu)(1-2 \nu) \sigma_{n}}{E(1-\nu)}=\frac{(1+\nu)(1-2 \nu) k_{a} \delta_{V}}{E(1-\nu) l_{35}} \\
\gamma=\frac{\delta_{H}}{d_{1}+d_{2}}=\frac{\tau_{n s}}{2 G}=\frac{(1+\nu) k_{s} \delta_{H}}{E l_{35}}
\end{array}\right\}
$$

From which the following formulae can be obtained.

$$
\left.\begin{array}{l}
k_{d}=\frac{E(1-\nu) l_{35}}{(1+\nu)(1-2 \nu)\left(d_{1}+d_{2}\right)} \\
k_{s}=\frac{E l_{35}}{(1+\nu)\left(d_{1}+d_{2}\right)}
\end{array}\right\}
$$

And $k_{r}$ can be determined as follows:

The rotational moment $M_{\varphi}$ of the spring $k_{r}$ is given by the following equation

$$
\begin{aligned}
M_{\varphi} & =\int_{-l_{35} / 2}^{l_{35} / 2} \frac{k_{d}}{l_{35}}(s \varphi) s d s=k_{r} \varphi \\
\therefore \quad k_{r} & =\frac{k_{a} l_{35}^{2}}{12}
\end{aligned}
$$

(v) Convergency test of beam and plate bending solutions

Fig. 7 shows the result of limit analysis of a beam clamped at both ends under a single concentrated 


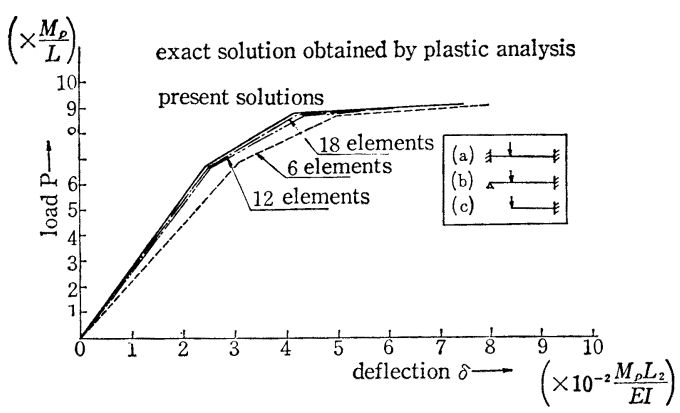

Fig. 7 Collapse load analysis of a clamped beam under a concentrated load

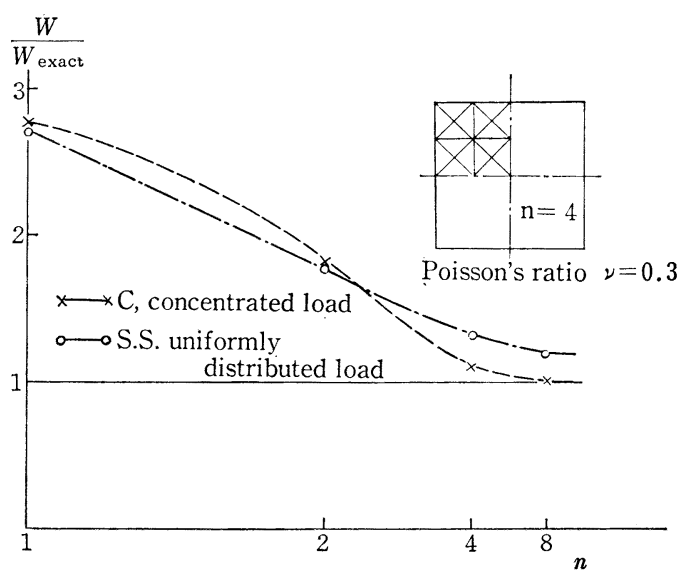

Fig. 8 Result of convergency tests

load.

Fig. 8 shows the result of convergency test of a rectangular plate bending solutions under a concentrated as well as uniformly distributed loads.

\section{Some Numerical Examples on the Limit Analyses of Plate Bending and Plane Strain Problems}

Currently a series of numerical studies on the applicability and efficiency of these new elements to analysis of structural problems have been made including linear and nonlinear problems (static, dynamic and instability). Due to space limitation, however, some examples on the collapse load analysis of square plates under lateral loads and limit analysis of the punch problem and elastoplastic analysis of a slit notch tensile specimen under tensile loading will be briefly discussed.

$(\alpha)$ Collapse load analysis of square plates under lateral load

Consider a square plate whose two neighbouring edges are simply supported while the other edges are made free, but supported by a column at the

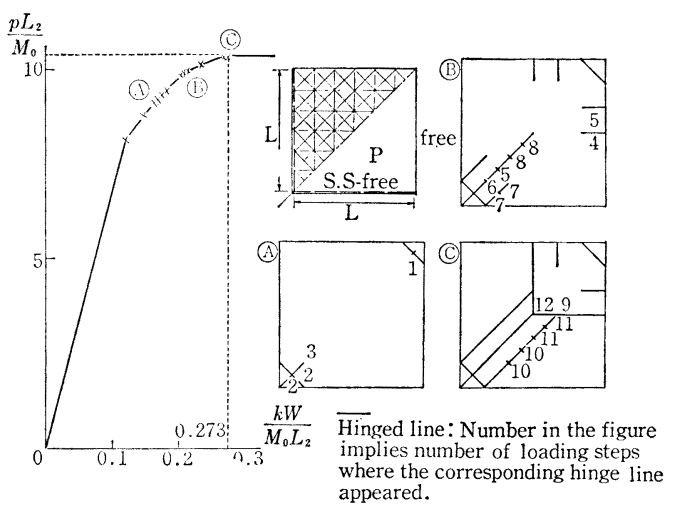

Fig. 9 Collapse load analysis of a rectangular plate under a uniformly distributed load

corner of two neighbouring free edges as shown in Fig. 9.

Using mesh division shown in the figure and adopting the standard load incremental procedure in the finite element analysis of inelastic problems, the load-deflection characteristics was searched under the assumption of uniformly distributed as well as a concentrated load. Results of analysis are shown in Figs. 9 and 10. Agreement between the present calculation and experiment made by other investigators ${ }^{2}$ was found to be extremely good.

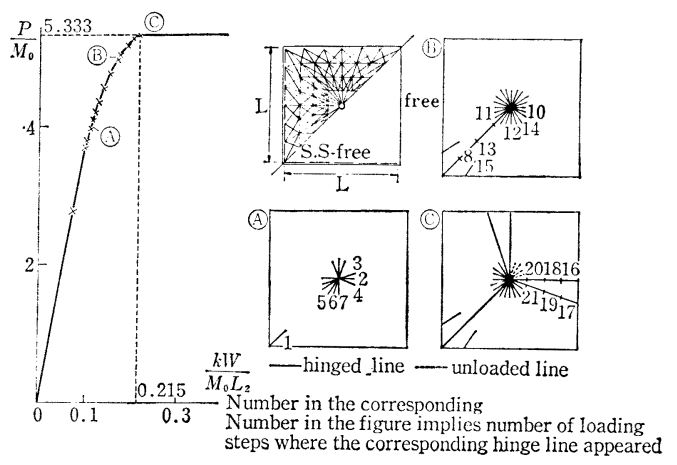

Fig. 10 Collapse load analysis of a rectangular plate under centrally concentrated load

Calculations were made by using the domestic computer "HITAC $8700-8800$ " which is approximately comparable to the IBM 360-195. CPU time for both cases was 10 seconds.

( $\beta$ ) Analysis of the punch problem

The punch problem of an elasto-plastic slab as shown in Fig. 11 is considered. This problem is a standard plane strain problem in plasticity and it was studied by many investigators for various cases of $h / b$ ratios using the so-called "slip line theory".

In the present analysis a given material is as- 
sumed to be ideal plastic and the maximum shearing stress theory is used as the yield criterion. Since the material is assumed incompressible after yielding, the plastic strain increment is purely shearing deformation. Analysis was made for three
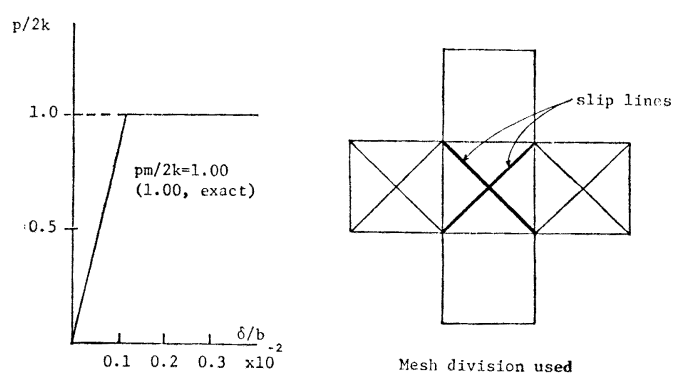

Fig. 11 Punch problem (i) $h / b=1$
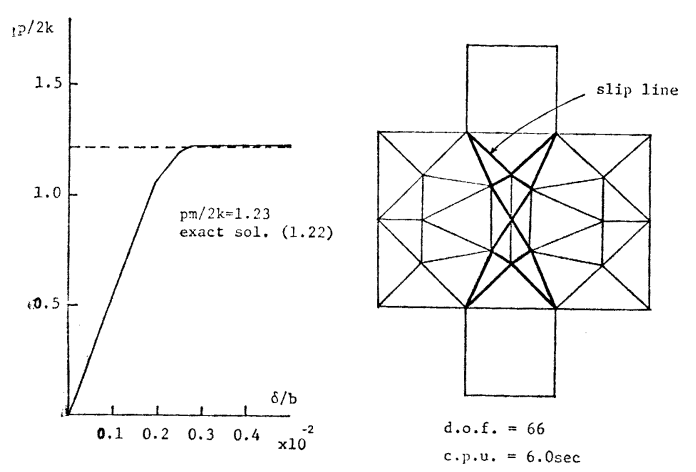

Fig. 12 Punch problem (ii) $h / b=2$ different cases of $h / b$ ratios as follows:
(i) $h / b=1$
(ii) $h / b=2$
(iii) $h / b \geqq 8.74$.

The load-deformation curves obtained, assumed mesh division and slip lines as well as computing time are shown in Figs 11,12 and 13.

It should be mentioned here that the rotational component $\theta_{i}$ was neglected in this analysis so that the size of stiffness matrices used was only $2 \times 2$ and yet the ultimate loads and slip lines obtained were in good agreement with the results obtained by previous authors.4)

$(\gamma)$ Elasto-plastic analysis of a slit notch tensile specimen

Elasto-plastic incremental analysis was made for the specimens as shown in Fig. 14 under the assumption of no rotational displacement and the maximum shearing stress theory. The result obtained was again in good agreement with the result of previous investigators ${ }^{5)}$.

\section{Conclusion}

A family of new elements especially suitable for nonlinear analysis of structural problems is proposed in this paper. Results of numerical analysis on some simple problems duly justified use of these elements for elasto-plastic structural analysis. Possible extension of this idea to analysis of three dimensional problems is obvious. Such extension and generalization of these elements is now under way. Extensive studies on the application of these models to limit analysis of structures, contact problems, plasticity, fracture mechanics, slope stability, etc. are now being planned.
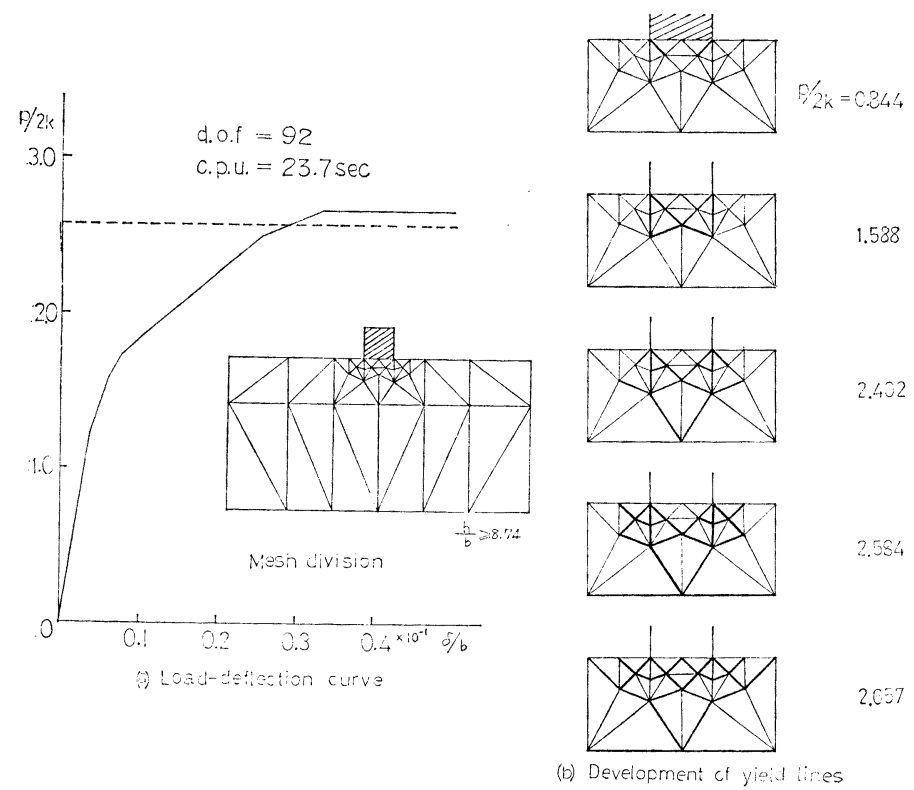

Fig. 13 Punch problem (iii) $h / b \geq 8.74$ 


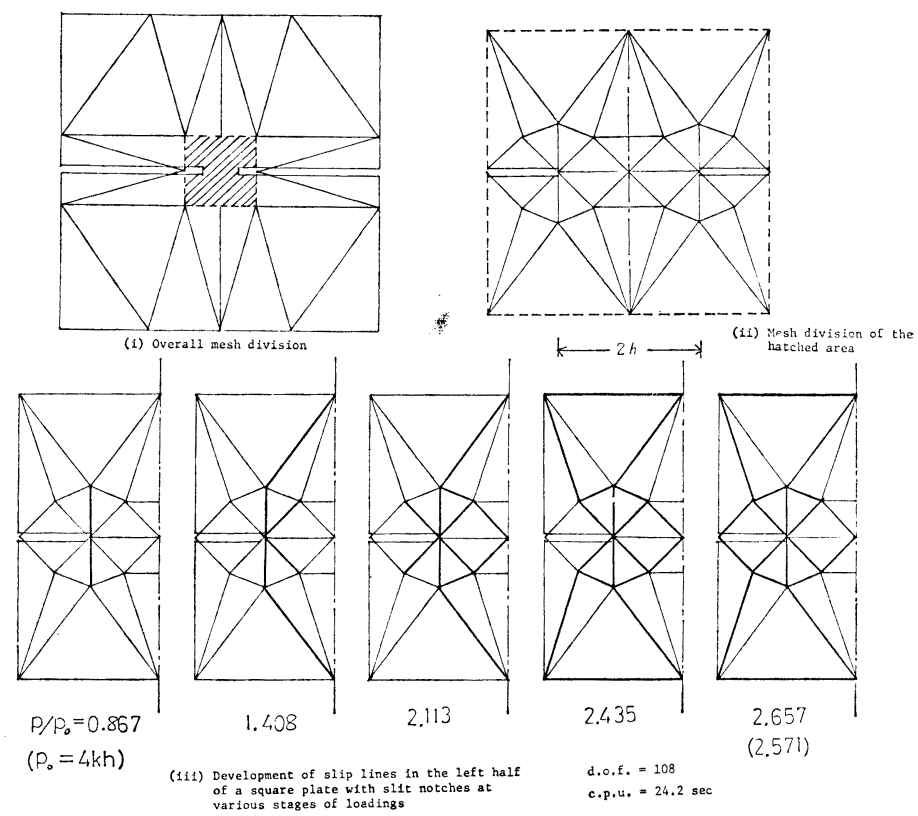

Fig. 14 Analysis of a square plate with slit notches under tensile liading (plane strain problem)

\section{Acknowledgements}

Firstly comments of the paper screeing committee is highly appreciated.

Secondly the author would like to express his sincere thanks to Messrs K. Kondou and Y. Toi, graduate students of the University of Tokyo. Mr. Kondou carried out collapse load analysis of square plates under lateral loading, while Mr. Toi conducted limit analysis of plane strain problems.

Effort of Miss Sueko Suzuki for typing the manuscript is also acknowledged.

\section{References}

1) T. Kawai and K. Kondou: New Beam and Plate Bending Elements in Finite Element Analysis, short communication of the "Seisan Kenkyu", Vol. 28, No. 9, September, 1976.

2) A.Sawczuk and T. Jaeger: Grenztragfahigkeits
Theorie der Platten, Springer-Verlag, Berlin, Gottingen, Heidelberg, 1963.

3) M. A. Save and C. E. Massonnet: Plastic Analysis and Design of Plates, Shells and'Disks, North-Holland Publishing Company, Amsterdam, London, 1972.

4) Yoshiaki Yamada: Plasticity, Nikkan Kogyo Shimbun Press., 1965. (in Japanese)

5) L. M. Kachanov: Foundations of the theory of plasticity, North-Holland, 1971.

6) H. Ford and J.M. Alexander: Advanced Mechanics of Materials, Longman, 1963.

7) R. Hill: The Mathematical Theory of Plasticity, Oxford at Clarendon Press, 1950.

8) P. G. Hodge, Jr.: Plastic Analysis of Structures, McGraw-Hill Book Co., Inc., 1959.

9) D.C. Drucker and J.R. Rice: Plastic Deformation in Brittle and Ductile Fracture, Engineering Fracture Mechanics, Vol. 1, 1970. 